\title{
Manifesto Internacional para a Promoção da Atividade Física no Pós-COVID-19: Urgência de uma Chamada para a Ação
}

\section{COMITÊ EDITORIAL \\ Antonio Carlos Bramante, Douglas Roque Andrade, Francisco José Gondim Pitanga, Lamartine Pereira da Costa, Luis Carlos de Oliveira, Luiz Guilherme Grossi Porto, Ma- ria Beatriz Rocha Ferreira, Markus Vinicius Nahas, Maurício Santos e Victor Keihan Rodrigues Matsudo.}

\section{AGRADECIMENTOS}

Fórum Agita Mundo: Detlef Dumon, Elio Antunes, Fiona Bullm, Kristin Belleson, Michael Pratt, Miguel Malo, Pedro Hallal, Vicki Lambert, Wendy Brown.

Fórum Rede de Atividade Física das Américas: Angel Javier, Maciste Habacuc, Maribel Parra, Mariona Violan, Nubia Ruiz Gomez, Oscar Diaz e Oscar Incarbone.

Fórum Agita São Paulo: Andréia Salvador, João Gabbardo dos Reis, João Pedro da Silva Júnior, José Luiz Gomes do Amaral e Natalia de Freitas Guerreiro Ferreira.

\section{CONTATO}

Victor Keihan Rodrigues Matsudo Centro de Estudos do Laboratório de Aptidão Física de São Caetano do Sul CELAFISCS

celafiscs.secretaria@gmail.com

Rua Santo Antônio, 50, Sala 505, São Caetano do Sul, São Paulo, Brasil.

CEP: 09521-160.

DOI

$10.12820 /$ rbafs. $25 \mathrm{e} 0175$

\section{(cc) BY}

Este trabalho está licenciado com uma Licença Creative Commons - Atribuição 4.0 Internacional.
"São convocados todos os cidadãos, governantes e dirigentes de entidades privadas a um amplo movimento em favor de uma vida mais ativa e saudável, para que possamos estar melhor preparados para a atual e futuras pandemias com características similares, ampliando-se os limites da solidariedade.

A pandemia exige que pensemos não só na segurança individual, mas também dos demais que nos cercam. Tão importante quanto a adoção de um estilo de vida mais ativo e a redução do comportamento sedentário no enfrentamento da pandemia da COVID-19, sobretudo, está na solidariedade entre todos, na busca por um mundo melhor, mais justo e mais saudável.”

Este manifesto é fruto de um esforço coletivo em prol de uma vida mais ativa, saudável e solidária para todos, proposto por profissionais e pesquisadores do Brasil e do exterior participantes do 43 Simpósio Internacional de Ciências do Esporte, realizado em São Paulo em outubro de 2020, promovido pelo CELAFISCS (Centro de Estudos do Laboratório de Aptidão Física de São Caetanos do Sul).

Este documento-referência procura sensibilizar e mobilizar os mais distintos grupos populacionais, órgãos governamentais, não governamentais e da iniciativa privada sobre a promoção de uma vida mais ativa, considerando os seus efeitos preventivos na mitigação de pandemias como a da COVID-19, especialmente quando realizada de forma intersetorial e multiprofissional.

Foram consideradas as orientações públicas da Organização das Nações Unidas (ONU), da Organização Mundial da Saúde (OMS) e do Colégio Americano de Medicina do Esporte (ACSM), incluindo artigos da edição temática da Revista Brasileira de Atividade Física e Saúde sobre Exercício Físico/Atividade Física e a COVID-19, assim como as publicações científicas sobre o tema, além de consultas a pesquisadores de reconhecimento local e internacional.

A essência deste documento prioriza a educação, valoriza a cultura local/ regional e propõe a redução de desigualdades às oportunidades de acesso aos bens públicos como caminho para uma vida mais ativa e saudável em comunidades mais solidárias. Destaca a importância da atividade física em relação à COVID-19.

A atividade física é um comportamento presente nas diversas dimensões da existência humana, devendo ser considerada como fundamental para a qualidade de vida em todas as faixas etárias. A alta prevalência de inatividade física traz consequências para a saúde individual e coletiva, com signifi- 
cativo impacto econômico e social. Para que todo o potencial da prática de atividade física seja alcançado, sua promoção, aliada à proposta de redução do comportamento sedentário, devem ser encaradas como um desafio de todos, principalmente em tempos de redução das oportunidades de prática, como na atual pandemia da COVID-19. Nunca os determinantes sociais da saúde e da qualidade de vida precisaram ser enfrentados de maneira tão ampla e urgente, com especial destaque a outro fator fundamental nas relações humanas e no combate às desigualdades e à intolerância: a solidariedade entre as pessoas, comunidades, cidades e nações.

Esforços articulados e sistêmicos são necessários para o enfrentamento a essas pandemias - COVID-19 e inatividade física. As ações mais efetivas têm considerado a cultura local, na perspectiva de uma dimensão global da crise, tal como em outras epidemias anteriores. Constatou-se que essas ações impactam diversos fatores associados à qualidade de vida, em particular nas relações interpessoais, renda familiar, padrões habituais de estudo, trabalho e lazer, eventos culturais e esportivos e na prática da atividade física.

Além dos diversos benefícios da atividade física para a prevenção e tratamento de doenças e na promoção da saúde, com destaque à atenção primária, deve-se enfatizar que o movimento é parte da natureza humana, fundamental para uma vida mais saudável, produtiva e plena.

As ações de promoção da atividade física e redução do comportamento sedentário devem educar, motivar e ampliar as oportunidades para escolhas bem informadas e qualificadas, explorando suas amplas possibilidades para um mundo melhor e mais solidário.

Lamentavelmente, uma política que associa a ampliação da prática de atividade física com a redução de um estilo de vida sedentário ainda é uma estratégia subvalorizada em nosso meio, tendo em vista os seus reais benefícios. Não vamos mais manter a atividade física como um "remédio secreto", diante de tantas e qualificadas evidências recomendando a sua promoção.

\section{Evidências científicas}

A importância da prática da atividade física para a saúde está amplamente sedimentada, com evidências para os sistemas cardiovascular, metabólico e imunológico, bem como para a saúde mental, com maior controle do estresse, ansiedade e depressão ${ }^{1-5}$. Mais recentemente, o estilo de vida ativo para a promoção da saúde passou a incluir, além da prática da atividade física, a redução do com- portamento sedentário. Assim, as novas recomendações têm associado a combinação desses dois fatores ${ }^{6-10}$.

Evidências recentes têm demonstrado que mesmo as atividades físicas de intensidade leve e de curta duração podem trazer benefícios à saúde. Qualquer movimento corporal é melhor do que nenhum e que mais é melhor que menos, onde todo passo conta para a prevenção de diferentes agravos à saúde e à sua promoção, resultando um estado de bem-estar geral. Portanto, os benefícios à saúde podem ocorrer em volumes bem menores do que os preconizados 150 minutos por semana ou 10 mil passos por $\mathrm{dia}^{11,12}$.

$\mathrm{O}$ isolamento físico decorrente das medidas sanitárias veio agravar o nível de sedentarismo mundial, motivando importantes pesquisadores e instituições, nacionais e internacionais, a chamarem a atenção para a necessidade de se promover a atividade física durante a pandemia ${ }^{13-18}$

A inatividade física já é considerada uma pandemia em si, responsável por mais de 5 milhões de mortes por ano ao redor do mundo ${ }^{19,20}$. A conjunção da pandemia da COVID-19 com a da inatividade física e da obesidade caracteriza um estado de sindemia, em que a interação entre elas resulta no aumento da prevalência de comorbidades, como obesidade, hipertensão, diabetes, câncer, doenças respiratórias e reumatológicas, principalmente em populações de baixa renda, o que afetaria ainda mais o risco de formas mais graves da COVID-19 ${ }^{21-24}$.

Há evidências de que houve uma redução drástica nos níveis de atividade física durante a pandemia ${ }^{25,26}$, apesar de recente publicação ter demonstrado um maior interesse na busca de informações sobre atividade física durante este período ${ }^{27}$.

A inatividade física pode reduzir os níveis de aptidão física, diminuindo a capacidade cardiovascular, força muscular, flexibilidade, equilíbrio, entre outras variáveis, contribuindo para o surgimento ou piora de comorbidades, como a obesidade, que podem agravar, ainda mais, os quadros clínicos de COVID-1928,29.

Evidências de pandemias virais anteriores indicaram que a atividade física influenciou na redução do agravamento do quadro clínico respiratório e na mortalidade ${ }^{30,31}$ e, de forma positiva, nos efeitos da vacinação, principalmente em idosos ${ }^{32-36}$. É plausível inferir que estes resultados poderiam ser transportados à realidade da COVID-1937,38. A atividade física leve e moderada tem mostrado respostas imunológicas em quadros de infecção, diminuindo marcadores pró-inflamatórios ${ }^{39}$. 
A literatura científica enfatiza, por outro lado, que não se deve recomendar atividade física intensa e de longa duração por levar à imunossupressão transitória, expondo as pessoas a maior possibilidade de contrair ou agravar a infecção viral ${ }^{40}$.

Estudos têm demonstrado a importância da prática de atividade física durante a pandemia em diferentes ambientes, especialmente dentro de casa e ao ar livre, com o devido distanciamento físico. Quanto aos centros de condicionamento físico, clínicas, clubes, escolas, entre outros ambientes fechados, será sempre necessário atender as medidas e as recomendações sanitárias, com a devida responsabilidade civil, os devidos princípios de solidariedade e ao desenvolvimento de uma cultura promotora de estilos de vida saudáveis ${ }^{13-18,38,41}$.

Esse conjunto de evidências conduz para uma chamada à ação.

\section{Urgência de uma Chamada para a Ação}

O cenário atual de pandemia e as evidências científicas revelam grandes desafios, dentre os quais, o de nos tornarmos ou nos mantermos fisicamente ativos, com toda segurança, respeitando-se, rigorosamente, as orientações comportamentais e ambientais que visam diminuir as chances de contágio pela COVID-19. É importante destacar que o quadro pós-pandêmico reduz as oportunidades da prática de atividade física, especialmente pelas pessoas com maior necessidade de isolamento físico, como idosos e portadores de doenças crônicas.

Neste contexto e à luz do atual conhecimento sobre os benefícios da atividade física e da adoção de um estilo de vida menos sedentário em relação a COVID-19, recomenda-se:

1) Buscar uma vida mais ativa e saudável, partindo da responsabilidade individual e social - eu, você e nós - além dos órgãos governamentais, não governamentais e instituições privadas, durante e após a pandemia, mesmo depois do surgimento de vacinas.

2) Promover esse estilo de vida amparado por políticas públicas e de organizações não governamentais, através de programas, projetos e ações, concretas e articuladas, em consonância com as demandas de desigualdades econômica e social, com foco na mobilização das novas gerações.

3) Priorizar uma vida mais saudável através da sinergia entre as diferentes políticas públicas, que enfatizem a importância da mobilidade ativa das pessoas, gerando efeito multiplicador sobre os demais benefícios de um estilo de vida ativo e sustentável, alicerçado na democratização de acesso aos serviços essenciais de saúde pública, saneamento básico e na universalização da educação de qualidade.

4) Fazer uso de estratégias inovadoras locais, em consonância com as diretrizes do Plano de Ação Global em Atividade Física 2018-2030 da OMS. A adoção dessa perspectiva "glocal" implica em priorizar a educação e reduzir desigualdades de acesso aos bens públicos no nível local, para se alcançar o objetivo global de se ter pessoas mais ativas em comunidades mais saudáveis e solidárias.

5) Focar no aumento dos estímulos e das oportunidades para o exercício de uma vida mais ativa no contexto da escola. Na retomada das atividades presenciais, será necessária uma profunda revisão do ambiente escolar, transformando-o em um polo promotor da atividade física combinado com a redução do comportamento sedentário, promovendo, dessa forma, a saúde ao longo da vida.

6) Buscar estratégias inovadoras para que as pessoas pratiquem suas atividades físicas em casa e nos ambientes abertos, respeitando-se todas as recomendações sanitárias de cada região, especialmente quando em locais fechados.

7) Incentivar o uso dos meios digitais para disseminar estratégias, recursos, modos e exemplos na promoção da atividade física e redução de comportamento sedentário, no sentido de levar as pessoas à adoção de um estilo de vida mais ativo e saudável.

8) Dar a devida atenção aos benefícios da atividade física moderada, realizadas de forma regular para o sistema imunológico, evitando-se as atividades físicas vigorosas em situações de exposição à COVID-19 ou às pessoas mais suscetíveis às suas formas mais graves.

9) Apoiar grupos sociais específicos nas diferentes manifestações da atividade física, com foco nos estudantes, nos trabalhadores, nos idosos, nas mulheres, nas pessoas com deficiências e outros grupos minoritários e vulneráveis.

10) Organizar as diferentes abordagens para a promoção e intervenção com foco nas atividades físicas segundo modelos do desenvolvimento sustentável e de gestão cooperativa por grupos de pessoas tendo por base o equilíbrio com os ambientes físico, social e econômico que os cercam.

11) Investir fortemente em políticas e ações que promovam a caminhada, o uso da bicicleta, a prática de es- 
porte, jogos e recreação mais ativa nos espaços públicos. 12) Promover uma atitude ativa no cotidiano. Mova-se mais e sente-se menos. Aumente seus passos diários. Preferencialmente, acumule 150 minutos ou mais por semana de atividade física moderada. Sempre que possível, substitua o tempo sentado por atividades físicas leves, como ficar de pé e se movimentar, lembrando que na promoção da saúde, todo movimento conta.

\section{Portanto, esta chamada para ação:}

"Convoca todos os cidadãos, governantes e dirigentes de entidades privadas a um amplo movimento em favor de uma vida mais ativa e saudável, para que assim possamos estar melhor preparados para a atual e futuras pandemias com características similares, ampliando-se os limites da solidariedade.

A pandemia exige que pensemos não só na segurança individual, mas também dos demais que nos cercam. Tão importante quanto a adoção de um estilo de vida mais ativo e a redução do comportamento sedentário no enfrentamento da pandemia da COVID-19, sobretudo, está na solidariedade entre todos, na busca por um mundo melhor, mais justo e mais saudável."

\section{Saiba mais}

Infográficos, versões em inglês e espanhol e outros materiais complementares podem ser consultados diretamente pelo link: https://celafiscs.org.br/manifesto-da-atividade-fisica/

\section{Referências}

1. Lin X, Alvim SM, Simoes EJ, Bensenor IM, Barreto SM, Schmidt MI, et al. Leisure Time Physical Activity and Cardio-Metabolic Health: Results From the Brazilian Longitudinal Study of Adult Health (ELSA-Brasil). J Am Heart Assoc. 2016;5(6).

2. Krinski K, Elsangedy H, Colombo H, Buzzachera C, Soares I, Campos W, et al. Physical exercise effects in the immunological system. Rev Bras Med. 2010;67(7):227-8.

3. Mammen G, Faulkner G. Physical activity and the prevention of depression: a systematic review of prospective studies. Am J Prev Med. 2013;45(5):649-57.

4. Haapanen N, Miilunpalo S, Vuori I, Oja P, Pasanen M. Association of leisure time physical activity with the risk of coronary heart disease, hypertension and diabetes in middleaged men and women. Int J Epidemiol. 1997;26(4):739-47.

5. Jonsdottir IH, Rodjer L, Hadzibajramovic E, Borjesson M, Ahlborg G, Jr. A prospective study of leisure-time physical activity and mental health in Swedish health care workers and social insurance officers. Prev Med. 2010;51(5):373-7.
6. van der Ploeg HP, Chey T, Korda RJ, Banks E, Bauman A. Sitting time and all-cause mortality risk in 222497 Australian adults. Arch Intern Med. 2012;172(6):494-500.

7. de Moraes AC, Carvalho HB, Rey-Lopez JP, Gracia-Marco L, Beghin L, Kafatos A, et al. Independent and combined effects of physical activity and sedentary behavior on blood pressure in adolescents: gender differences in two crosssectional studies. PLoS One. 2013;8(5):e62006.

8. Chau JY, Grunseit A, Midthjell K, Holmen J, Holmen TL, Bauman AE, et al. Cross-sectional associations of total sitting and leisure screen time with cardiometabolic risk in adults. Results from the HUNT Study, Norway. J Sci Med Sport. 2014;17(1):78-84.

9. Hamer M, Stamatakis E, Steptoe A. Effects of substituting sedentary time with physical activity on metabolic risk. Med Sci Sports Exerc. 2014;46(10):1946-50.

10. Pitanga FJG, Matos SMA, Almeida M, Patrao AL, Molina M, Aquino EM. Association between leisure-time physical activity and sedentary behavior with cardiometabolic health in the ELSA-Brasil participants. SAGE Open Med. 2019;7:2050312119827089.

11. Matsudo VKR, Beltran DCG, Guedes JS. Todo passo conta: Novas recomendações da atividade física. Diagnóstico e Tratamento. 2019; 24(1):21-4.

12. Porto LGG, Molina GE, Matsudo VK. Physical activity and the coronavirus pandemic: an urgent time to change the recommendation focus. Revista Brasileira de Atividade Física \& Saúde. 2020;25:1-5.

13. Chen P, Mao L, Nassis GP, Harmer P, Ainsworth BE, Li F. Coronavirus disease (COVID-19): The need to maintain regular physical activity while taking precautions. J Sport Health Sci. 2020;9(2):103-4.

14. ACSM. Staying physically active during the COVID-19 pandemic: American College Sports Medicine; 2020 [updated 01/27/2021. Available from: https://www.acsm.org/readresearch/newsroom/news-releases/news-detail/2020/03/16/ staying-physically-active-during-covid-19-pandemic.

15. Jiménez-Pavón D, Carbonell-Baeza A, Lavie CJ. Physical exercise as therapy to fight against the mental and physical consequences of COVID-19 quarantine: Special focus in older people. Prog Cardiovasc Dis. 2020;63(3):386-8.

16. Pitanga FJG, Beck CC, Pitanga CPS. Physical Activity And Reducing Sedentary Behavior During The Coronavirus Pandemic. Arq Bras Cardiol. 2020;114(6):1058-60.

17. Pitanga FJG, Beck CC, Pitanga CPS. Should Physical Activity Be Considered Essential During the COVID-19 Pandemic? International Journal of Cardiovascular Sciences. 2020;33(4):401-3.

18. Sallis JF, Pratt M. Multiple benefits of physical activity during the Coronavirus pandemic. Revista Brasileira de Atividade Física \& Saúde. 2020;25:1-5.

19. Pratt M, Ramirez Varela A, Salvo D, Kohl Iii HW, Ding D. Attacking the pandemic of physical inactivity: what is holding us back? British Journal of Sports Medicine. 2020;54(13):760.

20. Kohl HW, 3rd, Craig CL, Lambert EV, Inoue S, Alkandari JR, Leetongin $\mathrm{G}$, et al. The pandemic of physical inactivity: global action for public health. Lancet. 2012;380(9838):294-305.

21. Hall G, Laddu DR, Phillips SA, Lavie CJ, Arena R. A tale of two pandemics: How will COVID-19 and global trends in physical inactivity and sedentary behavior affect one another? Prog Cardiovasc Dis. 2020:S0033-620(20)30077-3. 
22. Luzi L, Radaelli MG. Influenza and obesity: its odd relationship and the lessons for COVID-19 pandemic. Acta Diabetol. 2020;57(6):759-64.

23. Carter SJ, Baranauskas MN, Fly AD. Considerations for Obesity, Vitamin D, and Physical Activity Amid the COVID-19 Pandemic. Obesity (Silver Spring). 2020;28(7):1176-7.

24. Pitanga FJG, Beck CC, Pitanga CPS. Inatividade física, obesidade e COVID-19: perspectivas entre múltiplas pandemias. Revista Brasileira de Atividade Física \& Saúde. 2020;25:1-4.

25. Fitbit. The Impact Of Coronavirus On Global Activity [Available from: https://blog.fitbit.com/covid-19-globalactivity/.

26. Tison GH, Avram R, Kuhar P, Abreau S, Marcus GM, Pletcher MJ, et al. Worldwide Effect of COVID-19 on Physical Activity: A Descriptive Study. Ann Intern Med. 2020;173(9):767-70.

27. Ding D, del Pozo Cruz B, Green MA, Bauman AE. Is the COVID-19 lockdown nudging people to be more active: a big data analysis. British Journal of Sports Medicine. 2020;54(20):1183.

28. Pinho CS, Caria ACI, Aras Junior R, Pitanga FJG. The effects of the COVID-19 pandemic on levels of physical fitness. Rev Assoc Med Bras (1992). 2020;66Suppl 2(Suppl 2):34-7.

29. Korakas E, Ikonomidis I, Kousathana F, Balampanis K, Kountouri A, Raptis A, et al. Obesity and COVID-19: immune and metabolic derangement as a possible link to adverse clinical outcomes. Am J Physiol Endocrinol Metab. 2020;319(1):E105-E9.

30. Wong C-M, Lai H-K, Ou C-Q, Ho S-Y, Chan K-P, Thach $\mathrm{T}-\mathrm{Q}$, et al. Is exercise protective against influenza-associated mortality? PloS one. 2008;3(5):e2108-e.

31. Siu E, Campitelli MA, Kwong JC. Physical activity and influenza-coded outpatient visits, a population-based cohort study. PLoS One. 2012;7(6):e39518.

32. Kohut ML, Cooper MM, Nickolaus MS, Russell DR, Cunnick JE. Exercise and psychosocial factors modulate immunity to influenza vaccine in elderly individuals. J Gerontol A Biol Sci Med Sci. 2002;57(9):M557-62.
33. Schuler PB, Leblanc PA, Marzilli TS. Effect of physical activity on the production of specific antibody in response to the 1998-99 influenza virus vaccine in older adults. J Sports Med Phys Fitness. 2003;43(3):404.

34. Kohut ML, Arntson BA, Lee W, Rozeboom K, Yoon KJ, Cunnick JE, et al. Moderate exercise improves antibody response to influenza immunization in older adults. Vaccine. 2004;22(17-18):2298-306.

35. Woods JA, Keylock KT, Lowder T, Vieira VJ, Zelkovich W, Dumich $\mathrm{S}$, et al. Cardiovascular exercise training extends influenza vaccine seroprotection in sedentary older adults: the immune function intervention trial. J Am Geriatr Soc. 2009;57(12):2183-91.

36. Wong GCL, Narang V, Lu Y, Camous X, Nyunt MSZ, Carre $\mathrm{C}$, et al. Hallmarks of improved immunological responses in the vaccination of more physically active elderly females. Exerc Immunol Rev. 2019;25:20-33.

37. Zbinden-Foncea H, Francaux M, Deldicque L, Hawley JA. Does High Cardiorespiratory Fitness Confer Some Protection Against Proinflammatory Responses After Infection by SARS-CoV-2? Obesity (Silver Spring). 2020;28(8):1378-81.

38. Sallis JF, Pratt M. Letter about Physical Activity Can Be Helpful in the Coronavirus Pandemic International Society of Behavioral Nutrition and PhysicalActivity2020 [updated 01/27/2021. Available from: https://www.isbnpa.org/index. php? $r=$ article/view\&id $=146$.

39. Abd El-Kader SM, Al-Jiffri OH. Impact of aerobic versus resisted exercise training on systemic inflammation biomarkers and quality of Life among obese post-menopausal women. Afr Health Sci. 2019;19(4):2881-91.

40. Rahmati-Ahmadabad S, Hosseini F. Exercise against SARSCoV-2 (COVID-19): Does workout intensity matter? (A mini review of some indirect evidence related to obesity). Obesity Medicine. 2020;19:100245.

41. Cortez ACL, Pitanga FJG, Almeida-Santos MA, Nunes RAM, Botero-Rosas DA, Dantas EHM. Centers of physical activities and health promotion during the COVID-19 pandemic. Rev Assoc Med Bras (1992). 2020;66(10):1328-34. 